\title{
Relaciones entre la teoría contemporánea del Estado soberano y la consolidación de la paz
}

\author{
Hideaki Shinoda
}

\begin{abstract}
The interventions of the international community in so-called failed states raise the question about the legitimacy of these actions in a world, in which the concept of state sovereignty is thought to have a particular importance. This paper examines the issue from a perspective of peacebuilding interventions and explains how nationalism, regionalism and globalism can foster or hinder the interventions. Then, the paper examines some institutional developments related to the issue, which have been made possible by introducing the concept of the responsibility to protect as one of the key concerns of the international community's activities.
\end{abstract}

\section{Introducción}

Este artículo pretende concentrarse en las interacciones entre nacionalismo, regionalismo y globalismo desde la perspectiva de la consolidación de la paz, especialmente para los llamados "Estados fallidos". El artículo también busca examinar cómo la consolidación de la paz ilustra la característica natural de la teoría del Estado soberano, al igual que examinar algunos documentos oficiales que hacen especial referencia a la idea de la "responsabilidad de proteger". Este documento argumenta que la idea de Estado soberano, desde la perspectiva de la consolidación de la paz para los Estados fallidos, está basada, en gran parte, en una teoría liberal de soberanía del Estado que contiene combinaciones complejas de nacionalismo, regionalismo y globalismo.

Los Estados soberanos no están simplemente apoyados por el nacionalismo en oposición al regionalismo o al globalismo, estos pueden obstaculizarlos o reforzarlos. El concepto de "Estados fallidos" muestra los momentos críticos de la soberanía del Estado, puesto que este sugiere que un Estado soberano no es una entidad estable, sino que puede ser trastornada o reformada. El nacionalismo, el regionalismo y el globalismo son elementos activos en la consolidación de la paz en los Estados fallidos, los cuales a menudo contienen actividades para 
restablecer los mecanismos disfuncionales del Estado. Los elementos del nacionalismo, regionalismo y globalismo pueden contribuir al avance de los procesos de consolidación de la paz en algunos casos; y pueden dificultar los esfuerzos en otros. Estos tres elementos forman parte del, aún más complicado, están entre los factores que hacen el proceso de consolidación de paz.

Este artículo argumenta que este complejo proceso de consolidación de la paz nos lleva a reconsiderar la característica de la teoría contemporánea de soberanía del Estado. Es incorrecto presuponer que la consolidación de la paz es sólo un intento de construcción nacional. También es incorrecto asumir que sus actividades son, finalmente, intervenciones de las fuerzas poderosas en contra de las más débiles. La consolidación de la paz simplemente no cuestiona o defiende al Estado soberano; ni cuestiona o defiende al nacionalismo, al regionalismo o al globalismo. Los tres elementos constituyen las complejas configuraciones actuales y sus actividades. El discurso sobre la responsabilidad de proteger, como base teórica de la consolidación de la paz para los Estados fallidos, determina cómo la sociedad internacional tiende a entender la soberanía del Estado en el siglo XXI.

La siguiente sección nos da una perspectiva básica de este artículo explicando la complicada relación entre la consolidación de la paz y la soberanía en relación con el nacionalismo, el regionalismo y el globalismo. La tercera sección contempla el discurso contemporáneo de la consolidación de la paz haciendo especial referencia a la "responsabilidad de proteger" para identificar la naturaleza característica de la teoría de la soberanía del Estado. Finalmente, este artículo concluye indicando las implicaciones de sus resultados para próximos estudios sobre la consolidación de la paz y la sociedad internacional.

\section{Nacionalismo, regionalismo y globalismo en la consolidación de la paz}

\section{El problema de los conceptos definidos geográficamente}

Cuando examinamos teóricamente el "globalismo, regionalismo y nacionalismo" tendemos a verlos como perspectivas o movimientos opuestos. Mientras analizamos las interacciones entre ellos, podemos terminar preguntándonos cuál de estos es ahora la fuerza más dominante o cuál lo será en el futuro. Este documento no cuestiona, necesariamente, tal perspectiva analítica, que es por supuesto muy útil e importante en muchos casos. Sin embargo, sí llama la atención al hecho de que estos tres paradigmas están definidos en campos geográficos. Si analizamos nuestro mundo contemporáneo sólo refiriéndonos a los conceptos definidos geográficamente, podríamos caer en la tentación de 
discutir sobre cuál unidad geográfica es la más fundamental en las relaciones internacionales. Pero el debate de las unidades no siempre es productivo. Aún si las fuerzas del globalismo, del regionalismo y del nacionalismo compiten entre sí, ellas también podrían hacerlo so pretexto de la misma bandera. Mientras algunos tipos de ellas forman un cierto conjunto de movimientos, otros tipos podrían constituir un grupo para desafiar al otro.

Los conceptos definidos geográficamente tienden a dar la impresión de que el movimiento de un nivel no contiene el carácter de movimiento en un nivel diferente. Cuando vemos un aumento de nacionalismo en el nivel Estadonación, tendemos a suponer que el movimiento no es ni regional ni global, pero esto no siempre es así. El nacionalismo puede surgir como resultado de cierto movimiento regional. Un típico ejemplo histórico es el aumento del nacionalismo en la época de la descolonización de Asia y África. El surgimiento de los sentimientos nacionalistas llevó a la independencia de numerosos Estados en las regiones, pero el proceso de descolonización fue avanzando como movimientos regionales. Asia y África experimentaron movimientos regionales para desafiar al legado colonial de los poderes europeos. El regionalismo facilitó o incluso produjo el nacionalismo en los recientes Estados independientes. Aún ahora, es muy frecuente el caso de que los países europeos mantengan el nacionalismo con un fuerte sentido de orgullo o identidad regional. No es contradictorio, en absoluto, tener una fuerte identidad nacional del país como un Estado miembro de la Unión Europea. El globalismo también puede ser una fuente de nacionalismo. El movimiento global por los derechos humanos, por ejemplo, podría dar a sus defensores afecto nacionalista por el país que lo apoya.

Los conceptos definidos geográficamente pueden coincidir en parte con ciertos valores políticos multidisciplinarios. Por ejemplo, el liberalismo como una de las más prominentes ideologías políticas en el mundo contemporáneo no está precisado por fronteras definidas geográficamente. Es la fuente del nacionalismo en países como los Estados Unidos. Hay un tipo de nacionalismo basado en el liberalismo como su fuente histórica inalienable. Algunas organizaciones regionales como la OTAN, OSCE, ${ }^{1}$ y la UE, que identifican al liberalismo como su valor indispensable, indican formas posibles de regionalismo basadas en el liberalismo. Claro está, hoy en día el liberalismo constituye un sistema de valores globalmente válido, aunque esto no significa que el "fin de la historia" ha llegado o que ningún ciudadano del mundo refute intervenciones hechas en nombre del liberalismo. 
Mientras destacamos la función peculiar de un cierto movimiento definido, podemos identificar interacciones entre movimientos categorizados de forma diferente. También podríamos insertar cierta perspectiva interdisciplinaria derivada de un marco conceptualizado de manera distinta. Podemos enriquecer nuestras discusiones de cualquier problema en la sociedad internacional refiriéndonos a su relevancia en el nacionalismo, el regionalismo o el globalismo.

\section{La consolidación de la paz en tres dimensiones}

"La consolidación de la paz" es también un buen ejemplo de las interacciones complejas de los movimientos ideológicos. A menudo se afirma que sus actividades son equivalentes a la "construcción nacional". Si bien es cierto que las actividades de la consolidación de la paz son con frecuencia dirigidas a establecer un mecanismo solido de nación-Estado, estimulando los sentimientos nacionales para movilizar élites y la solidaridad nacional. También es cierto que el nacionalismo puede trastornar los esfuerzos para reconstruir sociedades estables. Las emociones nacionalistas pueden fácilmente llevar a las personas de una sociedad local a perder la paciencia con el desarrollo lento y extenso de las actividades internacionales de la consolidación de la paz. El regionalismo es el factor clave en muchos de sus casos ya que las organizaciones regionales juegan un rol muy importante en la implementación de estas actividades. También es cierto que el regionalismo puede trastornar el manejo sensible de la reconstrucción de un Estado-nación. El globalismo, por su parte, es a menudo una dimensión crítica de la consolidación de la paz porque la comunidad internacional necesita la lógica del globalismo para justificar sus intervenciones. Finalmente, también es cierto que el globalismo puede perturbar sus esfuerzos de establecer y fortificar marcos de comunidades políticas.

Se ha dicho a menudo que los Estados fallidos simbolizan la necesidad de la consolidación de la paz. Sería útil enfocarse en el problema para identificar cómo el regionalismo, el nacionalismo y el globalismo se relacionan con ella. El nacionalismo puede fomentar la crisis de un Estado, no solamente cuando éste lleva a una nación al ejercicio militar contra otro Estado. El nacionalismo puede no siempre coincidir con las fronteras del Estado. El nacionalismo para un grupo de personas en Bosnia y Herzegovina (BIH), por ejemplo, podía ser una lealtad emocional hacia los serbios o hacia la república de Srpska (una unidad interna de $\mathrm{BIH}$ ), pero otros podrían tener un fuerte sentimiento nacionalista hacia la misma BIH. No es seguro si los afganos nacionalistas pretendían defender el gobierno nacional del presidente Karzai o criticar la presencia de las fuerzas internacionales respaldando al gobierno. No nos sorprendemos realmente de 
ver a los iraquíes atacando a las fuerzas de los Estados Unidos motivados por su sentimiento nacionalista, aunque con esto contribuyan más a la crisis de Irak.

El regionalismo también puede desestabilizar a un Estado. Es claro que el regionalismo es una de las causas de guerra tradicionalmente reconocidas, dada la historia de la Segunda Guerra Mundial. El regionalismo es relevante para algunos conflictos contemporáneos. El conflicto en el Medio Oriente ha ido evolucionando alrededor de una esclavitud emocional compartida por la gente de la región. Palestina no es solo el problema de alguien más para la mayoría de ciudadanos sino un problema de la región entera. La identidad regional tiende a ser un semillero del conflicto en áreas como el Cáucaso, donde las influencias entre rusos y europeos han sido balanceadas. En Timor Oriental, la influencia orientada hacia la región ejercida por Australia es chocante para quienes aprecian las tradiciones históricas derivada de los días de las colonia.

El globalismo es el mayor factor de conflictos armados en el mundo. El capitalismo global nunca ignora las áreas de conflicto donde se encuentran ricos recursos naturales. El capitalismo global extendió la guerra en Sierra Leona otorgándoles ganancias a fuerzas rebeldes que explotaban diamantes con fácil acceso al mercado global. Esto también aplica a los conflictos en la parte Oriental de la República Democrática del Congo así como a los de Sudán, donde la presencia de recursos naturales han agravado los peores casos de guerra del mundo. La economía del mercado global nunca deja de absorber los narcóticos ilegales de Afganistán, aún cuando esto debilitaría substancialmente al gobierno central. La circulación global de armas, de humanos, de ideas fundamentalistas, etc., puede beneficiar a quienes quieren continuar la guerra.

Los tres movimientos no son irrelevantes en la mayoría de casos de Estados fallidos. Por el contrario los elementos de nacionalismo, regionalismo y globalismo generalmente se encuentran presentes cuando un Estado deja de funcionar apropiadamente. Ellos no compiten entre sí en estos casos, sino que más bien podrían ejercer conjuntamente impactos negativos sobre el desarrollo del proceso de descomposición de los mecanismos de Estado. Sin embargo, estos tres movimientos también podrían contribuir al desarrollo de la consolidación de la paz reestableciendo los mecanismos estatales en Estados fallidos. El nacionalismo es casi un elemento indispensable de reconstrucción de un Estado unificado. Sin el sentimiento de nacionalismo y el sentido de identidad sería muy difícil constituir una comunidad política estable en el marco de un Estado-nación. Teóricamente, una sociedad en paz puede ser construida sin atraer al elemento nacionalista. Sin embargo es muy difícil crear tal sociedad sin recurrir al internacionalmente reconocido marco de Estado-nación. Es por 
eso que la consolidación de la paz es a veces considerada como un equivalente de la construcción nacional. El nacionalismo es un motor emocional para la construcción de una sociedad en paz según el amplio marco compartido de la comunidad política, el Estado-nación.

El regionalismo es una de las más útiles herramientas en la construcción de paz. Cuando una nación no tiene la capacidad suficiente de mantener la paz por sí misma, es muy normal que a los países vecinos se les pida movilizar recursos. El regionalismo ha cambiado las áreas de actividades de las organizaciones regionales como la OSCE y la NATO, quienes no dudan en conducir actividades de consolidación de la paz. La OSCE tiene muchas oficinas nacionales en Europa y sus alrededores para facilitar el proceso de construcción de paz en países como Bosnia y Herzegovina. En África los roles de la Unión Africana (UA) y también de los países pertenecientes a ECOWA ${ }^{2}$ han sido recientemente expandidos al área de operaciones de paz. El reciente despliegue de tropas de paz (peacekeepers) de la UA a Darfur en Sudán es una de las más destacadas iniciativas hechas por una organización regional. El regionalismo es una perspectiva indispensable, especialmente cuando el conflicto armado surge con cierto fondo político regional. Por ejemplo, no es conveniente considerar separadamente los casos de Rwanda, Burundi y la República Democrática del Congo, que constituyen la llamada Región de Grandes Lagos. En estos casos cada conflicto en cada país tiene que ser analizado desde la perspectiva de la región en su totalidad.

El globalismo es ciertamente un elemento indispensable para el éxito de la construcción de paz. La solidaridad global es requerida para erradicar las causas del conflicto armado, cuando la consolidación de la paz necesita ser reforzada por el control global de armas, materiales, productos, recursos naturales, etc. Las reglas de las operaciones de paz tienen que ser universalmente reconocidas. En los procesos de construcción de paz, por ejemplo, el trato a los criminales de guerra es uno de los más comunes y serios inconvenientes. Aún cuando cada caso de construcción de paz tiene su propia manera distinta de implementar sus políticas, debe haber un conjunto de reglas comunes para manejar el problema, como una ley humanitaria internacional y una ley de derechos humanos internacional. Además, y en primer lugar, para sostener la voluntad política de la comunidad internacional en la consolidación de la paz, debe haber un interés global en la solución de conflictos armados.

Los elementos de nacionalismo, globalismo y regionalismo existen tras los bastidores de los Estados fallidos. También existen en los procesos de consoli- 
dación de paz, y ninguno puede ser considerado como la única razón dominante en la creación de Estados fallidos o en la consolidación de la paz. Esta es la importancia analítica de introducir estos tres elementos.

\section{La consolidación de la pary la soberanía del Estado}

La consolidación de la paz tiene su particular orientación hacia la soberanía del Estado. Cuando trata con los Estados fallidos, la comunidad internacional implementa actividades de construcción de paz con el objetivo de restablecer un Estado soberano. La creación de un Estado soberano estable es el supuesto objetivo de la construcción de paz. Esta no es una conclusión teóricamente inevitable. Sin embargo, es común entender que el restablecimiento del Estado soberano es el objetivo de la consolidación de la paz.

Esta meta tiende a ser llevada a cabo a pesar de la relación de la consolidación de la paz con el nacionalismo, el globalismo y el regionalismo. Estos tres elementos están presentes de todas maneras en la aparición de un Estado fallido y en el desarrollo de la consolidación de la paz. El Estado soberano no es producto del nacionalismo, ni es creado solo por el globalismo o el regionalismo. Está fomentado y limitado por los tres elementos, actuando conjuntamente o separadamente de maneras compleja.

Por lo tanto, es incorrecto suponer que la construcción de la paz es un asunto del nacionalismo. El hecho de que la consolidación de la paz pretenda establecer un Estado soberano no significa que aquellos que están comprometidos en las actividades de construcción de paz se conviertan automáticamente en agentes del nacionalismo. Es incorrecto pensar que la construcción de paz es una fuerza externa en contra de actores de la sociedad local. Ninguna actividad de la consolidación de la paz puede surtir efecto sin la participación de un actor local. Al final, la consolidación de la paz siempre termina relacionada con la promoción de la capacidad de construcción de la paz duradera en una sociedad local. De esta manera, no sería apropiado la pregunta cuál de los elementos se volverá más dominante cuando la consolidación de la paz sea completamente satisfactoria. No hay una respuesta a esta pregunta, ya que la construcción de la paz al restablecer el Estado soberano en un previo Estado fallido toma lugar sin perjudicar a los tres elementos.

Pero, ¿es realmente cierto que el globalismo se está reconciliando con el Estado soberano? ¿Es realmente posible avanzar en la construcción de la paz restableciendo el Estado soberano sin privilegiar a ninguno de los tres elementos de nacionalismo, globalismo o regionalismo? La respuesta afirmativa a estas preguntas, al menos hasta cierto punto, será dada después de examinar la 
teoría contemporánea del Estado soberano siendo aplicada en el campo de la construcción de paz.

\section{La consolidación de la paz como el momento crítico de la sobe- ranía del Estado}

Teoría política de la consolidación de la paz.

Para examinar la relación entre la consolidación de la paz y el Estado soberano es conveniente reflexionar sobre el punto de vista de la teoría política. Esto nos da los fundamentos teóricos y nos permite explicar cómo la consolidación de la paz se relaciona con la soberanía del Estado.

Existen múltiples maneras de teorizar la soberanía del Estado. ${ }^{3}$ No es exagerado decir que el número de teorías posibles sobre la soberanía es casi ilimitado. Pero al mismo tiempo es importante identificar el tipo de inclinaciones políticas que dominan las teorías de la soberanía en una época particular. En cada época siempre hay modos dominantes de pensamientos políticos de acuerdo al ambiente político de los tiempos, aun si es todavía cierto que una sola tendencia no excluye a las demás en ningún evento. Es evidente que, en la época de la post Guerra Fría, la teoría de la soberanía basada en ideas comunistas es anticuada. Es también seguro decir que la teoría de la soberanía basada en la teoría política de la democracia liberal tiene una influencia predominante en el mundo contemporáneo -aunque esto no invalida a otras formas de pensamiento político en el mundo-, dado el hecho de que la mayoría de Estados industrializados la han adoptado como fundamento constitucional de su sistema político y han formado alianzas políticas, militares y económicas con los Estados de ideas similares. Uno se pregunta si podemos encontrar una sola explicación para la democracia liberal, dada la historia tan amplia y diversa que tiene. Este artículo argumenta que, teniendo en cuenta el grado de influencia a nivel internacional, es crucial analizar la tradición anglo-americana de democracia liberal, la cual puede ser considerada como la teoría política más influyente del mundo contemporáneo.

La teoría anglo-americana de democracia liberal tiene sus orígenes teóricos en la teoría de contrato social en los siglos XVII y XVIII. Las revoluciones en este periodo en Gran Bretaña y Estados Unidos establecieron los más antiguos sistemas de nuestro mundo contemporáneo. Thomas Hobbes en su Leviatán, publicado en 1651, argumentaba que para establecer un poder común que aca-

3 Véase Shinoda, Hideaki, Re-examining State Sovereignty: From Classical Theory to the Global Age, London, Macmillan, 2000. 
bara con el "estado de naturaleza" se requiere un pacto de cada hombre con cada hombre ...

\begin{abstract}
[...] de tal manera que cada uno de ellos pueda decir al otro: 'Yo autorizo y otorgo mi derecho de gobernante a este hombre o a esta asamblea, con la condición de que tú des el mismo derecho a él y lo autorices para actuar en todo de esta manera' Este hecho, la multitud unida en una persona, se llama Common-Wealth, en latín Civitas". Esta es la generación del grandioso Leviatán, o mejor, (para decirlo más reverentemente) del Dios Mortal, a quien debemos debajo del Dios Inmortal nuestra paz y protección. ${ }^{4}$
\end{abstract}

Hobbes empezó su teoría con los derechos naturales de los individuos, que simbolizó la tendencia liberal de esta. Declaraba que para mantener los derechos individuales en la realidad, una comunidad política necesitaba un poder soberano.

La palabra "autorizo" aquí es importante. La autorización autónoma es una condición de una república independiente (self-suffient commonwealth) e indica el Estado autónomo de la teoría liberal. Lo simbólico es que el "Dios Mortal" es al mismo tiempo un "hombre artificial", "en la que la soberanía es un alma artificial, que da vida y movimiento al cuerpo entero". ${ }^{5}$ La teoría de autorización de Hobbes hace posible el surgimiento del Estado autónomo.

Apareció poco después de Hobbes, en la época de la Revolución Gloriosa (1688), el campeón de la tradición anglo-americana de la teoría liberal, John Locke. ${ }^{6}$ Locke no discurrió totalmente sobre la teoría de soberanía, pero su posición sobre ésta es evidente en su texto Dos tratados sobre el gobierno civil.? Fue su crítica a la noción absoluta de soberanía la que lo llevó a la creación de dos poderes supremos.

Locke desarrolló una teoría de una sociedad autónoma política estableciendo dos "poderes supremos". Por una parte, Locke afirmó que aún después de acceder a formar una comunidad, "todavía queda un poder supremo en la gente". Según él, "la comunidad retiene perpetuamente un poder supremo". Los individuos nunca renuncian a su derecho de apelación al "cielo", a saber, el

4 Thomas Hobbes, Leviathan, edited by C.B. Macpherson, London, Penguin Books, 1985, (publicado originalmente en 1651), p. 227.

5 Ibid., p.81.

6 El contraste entre la "soberanía según la tradición lockeana”, que incluye a George Lawson y los federalistas americanos, y la "soberanía según Bodin, Hobbes y Rousseau”, se discute, por ejemplo, en: Mostov, Julie, Powver, Process, and Popular Sovereignty, Philadelphia, Temple University Press, 1992, pp. 52-72.

7 Locke, John, Two Treatises of Government, Cambridge, Cambridge University Press, 1960, pp. 326-7. 
derecho de resistencia y revolución, en caso de abuso de poder gubernamental. ${ }^{8}$ Por otra parte, señaló que aunque la comunidad "es siempre el poder supremo, pero no bajo la consideración de alguna forma de gobierno, porque este poder de la gente nunca puede tener lugar hasta que el gobierno sea disuelto".

Evitando la palabra soberanía, Locke declaró los dos poderes supremos. Esta teoría es posible, porque los diferenció entre "la disolución de la sociedad" y la "disolución del gobierno". ${ }^{10}$ Un poder supremo reina en la sociedad mientras el otro representa el poder supremo del gobierno. Esta "distinción entre el poder constituido y el ordinario" fue el fundamento de las revoluciones civiles, puesto que el abuso del poder gubernamental resultaría ahora en invocar al otro poder supremo popular. ${ }^{11}$ Esta distinción lleva a la premisa más importante de la constitucionalidad moderna: la distinción entre la autoridad constitutiva y el poder ordinario, es decir, entre las reglas constitucionales y las leyes ordinarias. Esta fue la lógica utilizada para legitimar la Revolución Gloriosa, y a la que también recurrieron posteriormente los revolucionarios de las trece colonias inglesas en Norteamérica.

Si presentamos la teoría de Estado de Locke en términos contemporáneos, encontraremos a los dos poderes supremos en la teoría liberal de soberanía. El ejecutor del poder gubernamental supremo debe proteger los derechos fundamentales de los individuos, puesto que la gente, como un conjunto, mantiene el poder supremo de autorización. Ambos poderes supremos son parte de la teoría integrativa de soberanía en la teoría política de la democracia liberal. Este es un concepto de soberanía en los Estados constitucionales contemporáneos.

Esta concepción liberal de soberanía también cultiva el fundamento de la teoría política de consolidación de la paz. ${ }^{12} \mathrm{El}$ poder gubernamental podría, a veces, no estar dispuesto o no ser capaz de proteger a los ciudadanos, o podría no ser suficientemente capaz de protegerlos adecuadamente. Este es el caso de un Estado fallido. Bajo tales circunstancias, algunos tipos de actividades de consolidación de la paz, e incluso intervenciones humanitarias, podrían ser justificadas. La comunidad internacional puede fomentar y ayudar al gobierno a proteger a los ciudadanos. Esta es una razón teórica que justifica las actividades internacionales de consolidación de la paz.

8 Ibid., p. 445.

9 Ibid., p. 385.

10 Ibid., p. 424.

11 Franklin, Julian H., John Locke and the Theory of Sovereignty: Mixed Monarchy and the Right of Resistance in the Political Thought of the English Revolution, Cambridge: Cambridge University Press, 1978, p. 124.

12 En lo relacionado a los aspectos operacionales creados por la influencia de la democracia liberal en la construcción de paz, véase: Paris, Roland, At war's end: building peace after civil conflict, Cambridge, Cambridge University Press, 2004. 
La lógica subyacente a la consolidación de la paz está teóricamente fundada sobre el concepto liberal de soberanía; esto explica por qué no contradice al Estado soberano. Mejor dicho, por paradójico que pueda ser, la teoría de soberanía es el mismo fundamento de la consolidación de la paz. La soberanía entendida en un marco constitucional justifica e incluso exige la consolidación de la paz por el bien de los derechos de los ciudadanos y el bienestar de la gente. Si el ejecutor del poder supremo no está dispuesto o no puede practicar su poder adecuadamente en nombre del poder supremo constitutivo de la gente, la teoría de soberanía requiere y justifica la ayuda externa en nombre de la consolidación de la paz.

\section{La responsabilidad de proteger}

Las implicaciones de la teoría liberal de soberanía es enorme para las discusiones que cubren desde la intervención humanitaria hasta la consolidación de la paz. La teoría liberal implica que no todas las intervenciones en Estados soberanos violan la soberanía. Aún cuando una intervención repudia a cierto poder gubernamental, esta podría no ser una violación a la soberanía. Si se ha abusado del poder gubernamental, el orden constitucional se ve destruido. Entonces se requeriría y justificaría una acción revolucionaria para reemplazar a quien ejerce el poder gubernamental en nombre del poder supremo del pueblo. Este es un típico criterio de justificación de la intervención humanitaria contemporánea. También necesitamos darnos cuenta de que esta lógica justifica las actividades de consolidación de la paz.

Es muy importante en este contexto ver el argumento del Reporte de la Comisión Internacional de Intervención y Estado de Soberanía (ICISS) organizada por el gobierno canadiense, publicado en diciembre de 2001 bajo el título La Responsabilidad de Proteger. ${ }^{13}$ Su publicación fue un evento muy importante entre académicos y profesionales del área, aunque la conmoción que produjeron los ataques del 11 de septiembre y la subsecuente "guerra contra el terrorismo" opacaron al documento inmediatamente después de su publicación. Aun así, no deberíamos subestimar su significado considerando el incremento gradual de su impacto en la sociedad internacional.

La ICISS fue precedida por Gareth Evanth, antiguo ministro de asuntos internacionales de Australia y para entonces presidente de la ONG international Crisis Group, y Mohamed Sahnoun de Algeria, consejero especial del Secretario General de las Naciones Unidas. Uno de los dos canadienses presentes entre los

13 Report on the International Commission on Intervention and State Sovereignty, The Responsibility to Protect, Ottawa, International Development Research Centre, 2001. 
12 miembros fue Michael Ignatieff, conocido escritor sobre temas relacionados con intervenciones humanitarias y la consolidación de la paz. ${ }^{14}$

El argumento básico para la "responsabilidad de proteger" está alineado con la misma tradicional teórica liberal de soberanía. Los dos principios básicos de su argumento son los siguientes:

A. La soberanía estatal implica responsabilidad, y la responsabilidad primordial de la protección de su pueblo radica en el Estado mismo.

B. Cuando la población está sufriendo daños serios, como resultado de la guerra interna, la insurgencia, la represión o el fracaso del Estado, y el Estado en cuestión no tiene la voluntad o la capacidad impedir o ponerle fin esta situación, el principio de la no intervención le da paso a la responsabilidad internacional de proteger.

Estos dos principios representan el valor central del liberalismo desde la época de John Locke. La soberanía es un principio inalienable de orden legal, político y social, tanto a nivel nacional como internacional. Pero esta noción de soberanía contiene el principio de responsabilidad entre los dos poderes supremos. El ejecutor de la soberanía es responsable de proteger los derechos fundamentales de los individuos. Una vez se pruebe que el Estado o gobierno no está dispuesto o no es capaz de asumir esta responsabilidad, el máximo poder supremo reasume la soberanía. Consecutivamente, el pueblo soberano está habilitado para la "apelación al cielo" o a recurrir incluso a la revolución. Esta es la teoría de soberanía en la tradición angloamericana de la democracia liberal. Podemos añadir que en el contexto de la sociedad contemporánea internacional, el hecho de "apelación al cielo" podría justificar la intervención humanitaria para ayudar a las personas a protegerse a sí mismas en caso de negligencia o abuso grave del poder gubernamental. Esta es la teoría de soberanía de la escuela de la "responsabilidad de proteger". ${ }^{14}$ La ICISS afirma que "los fundamentos de la responsabilidad de proteger, como un principio guía para la

14 Pensar la soberanía como responsabilidad, una forma que está siendo cada vez más reconocida en la práctica estatal, tiene significado en tres sentidos. En primer lugar, esto implica que las autoridades estatales son responsables de las funciones de proteger la seguridad y las vidas de los ciudadanos y de la promoción de su bienestar. En segundo lugar, significa que las autoridades políticas nacionales son internamente responsables ante los ciudadanos e internacionalmente ante la comunidad internacional a través de la ONU. Y en tercer lugar, esto significa que los agentes del Estado son responsables por sus acciones, es decir, son responsables por sus actos de comisión y omisión. El llamado a pensar la soberanía en estos términos se ve reforzado por la creciente influencia de las normas internacionales de derechos humanos y del discurso del concepto de seguridad humana". Report on the International Commission on Intervention and State Sovereignty, The Responsibility to Protect, Ottawa, International Development Research Centre, 2001 p. 13. 
comunidad internacional de Estados, descansan en (1) la obligación inherente en el concepto de soberanía; (2) la responsabilidad del Consejo de Seguridad, bajo el artículo 24 de los estatutos de las Naciones Unidas, que velan por el mantenimiento de la paz y la seguridad internacional; (3) las obligaciones legales específicas cobijadas por declaraciones de derechos humanos y de protección humana, convenios, tratados y la ley humanitaria nacional e internacional; y (4) las prácticas en desarrollo de los Estados, las organizaciones regionales y el mismo Consejo de Seguridad".

Posteriormente la ICSS proclama que "la responsabilidad de proteger abarca, a saber, tres responsabilidades especificas".

A. La responsabilidad de prevenir: atender las causas de origen y las causas directas del conflicto interno y otras crisis creadas por el hombre que ponen a la población en peligro.

B. La responsabilidad de reaccionar: responder a situaciones de necesidad humana contundente con medidas apropiadas, que podrían incluir medidas coactivas como sanciones, juicios internacionales y, en casos extremos, intervención militar.

C. La responsabilidad de reconstruir: proporcionar, especialmente después de una intervención militar, asistencia total con recuperación, reconstrucción y reconciliación, identificando las causas de los peligros que la intervención ha intentado detener o evitar.

En resumen, La ICISS insiste que la prevención del conflicto, la intervención humanitaria y la consolidación de la paz son asuntos de responsabilidad de la comunidad internacional, mientras que la responsabilidad inicial primaria es "inherente en el concepto de soberanía".

\section{La consolidación de la pazy la soberanía como responsabilidad}

$\mathrm{El}$ argumento a favor de la responsabilidad de proteger (RTP) ${ }^{15}$ fue aprobado por Canadá, patrocinador de la ICISS, y de los otros miembros de la llamada Red de Seguridad Humana, liderada por Canadá, que puede ser considerada como una coalición de Estados que reconocen la importancia del argumento pro RTP. Al principio, sin embargo, su influencia se vislumbraba limitada, ya que parecía haber una brecha entre la ICISS y la corriente principal de la comunidad internacional. Esto ha cambiado desde la publicación del reporte Un 
mundo más seguro: Nuestra responsabilidad compartida (2004) del panel de alto-nivel sobre amenazas, retos y cambios del Secretario General, comisionado por el Secretario General de la ONU, Kofi Annan. ${ }^{16}$

Mientras que la "responsabilidad de proteger" fue una respuesta a los debates internacionales sobre intervención humanitaria después de la crisis de Kosovo, Nuestra responsabilidad compartida es una respuesta al debate internacional sobre las nuevas amenazas de seguridad después de la guerra de Irak. Lo interesante es que el panel de alto nivel incluía a Gareth Evans, co-presidente de la ICISS, entre otros dieciséis miembros. Por consiguiente es claro suponer que el título Nuestra responsabilidad compartida hace referencia a la RTP.

En realidad, Nuestra responsabilidad compartida menciona a la responsabilidad de proteger. Este es el concepto clave cuando el panel de alto nivel discute el asunto de seguridad colectiva. El panel expone que:

Los sucesivos desastres en Somalia, Bosnia y Herzegovina, Rwanda, Kosovo y ahora Darfur, Sudán, han centrado su atención, no en las inmunidades del Estado soberano, sino en sus responsabilidades hacia su propio pueblo y hacia la amplia comunidad internacional. Hay un creciente reconocimiento a la cuestión, no del derecho de intervenir de cualquier Estado, sino del derecho de proteger de cada Estado cuando se trata de un pueblo padeciendo una catástrofe evitable - asesinatos y violaciones masivas, limpieza étnica por expulsión forzada y terror, hambruna premeditada y amenaza de enfermedades. Y existe una aceptación creciente de que mientras el gobierno soberano tiene la responsabilidad primaria de proteger a sus propios ciudadanos de estas catástrofes, cuando éste no está dispuesto o es incapaz de hacerlo, la responsabilidad debe ser adoptada por la amplia comunidad internacional - abarcando en esto un espectro continuo de activididades que incluyen la prevención, la reacción a la violencia, si es necesario, y la reconstrucción de sociedades destrozadas. El enfoque primario debe asignarse a cooperar en el cese de la violencia a través de mediaciones y otras herramientas, y a la protección de la población a través de medidas como el despliegue de misiones humanitarias, de derechos humanos y policiales. La fuerza, si se hace necesario su uso, debe ser ejercida como último recurso. Apoyamos la norma emergente de que existe una responsabilidad de proteger de carácter internacional y colectivo, que puede ser ejercida como último recurso tras autorización del Consejo de Seguridad para 
implementar la intervención militar, en caso de genocidio y otro tipo de exterminio a larga escala, limpieza étnica o violaciones graves de la ley humanitaria internacional, siempre y cuando el gobierno soberano haya probado su inhabilidad e impotencia para prevenirlas. ${ }^{17}$

Este es el momento para que la RTP sea incluida en un documento oficial de la ONU, mientras que Nuestra responsabilidad compartida es simplemente un reporte de la comisión independiente organizada por el Secretario General. Esta clara afirmación sobre la lógica de la RTP registra una nueva etapa de la teoría de la soberanía en la sociedad internacional. La consolidación de la paz está reconocida como un asunto de RTP en el reporte del panel, el cual estipula que:

[...] existe una aceptación creciente de que mientras el gobierno soberano tiene la responsabilidad primaria de proteger a sus propios ciudadanos de estas catástrofes, cuando éste no está dispuesto o es incapaz de hacerlo, la responsabilidad debe ser adoptada por la amplia comunidad internacional - abarcando en esto un espectro continuo de activididades que incluyen la prevención, la reacción a la violencia, si es necesario, y la reconstrucción de sociedades destrozadas.

Teniendo esto en consideración, es de gran importancia el hecho de que el nuevo órgano de las Naciones Unidas, la Comisión para la Consolidación de la Paz (PBC), ${ }^{18}$ fuera recomendada por el panel. La idea de la PBC, y también el acompañamiento de la Oficina de Apoyo de la Consolidación de la Paz (PBSO), ${ }^{19}$ fueron aprobadas por Kofi Annan en su "Concepto más amplio de la libertad" de 2005, con algunas modificaciones en el ámbito de las actividades de la PBC y su estructura organizacional. ${ }^{20}$ En la Cumbre Mundial de 2005 se decidió el establecimiento de la PBC Las resoluciones simultáneamente adoptadas por el Concejo de Seguridad y por la Asamblea General en diciembre 20 de 2005 decidieron la instauración de la PBC y de la PBSO. ${ }^{21}$

18 Siglas en inglés de Peacebuilding Commission (N. del ed.).

19 Siglas en inglés de Peacebuilding Support Office (N. del ed.).

20 "Integrated and Coordinated Implementation of and Follow-up to the Outcomes of the Major United Nations Conferences and Summits in the Economic, Social and Related Fields: Follow-up to the Outcome of the Millennium Summit: In Larger Freedom: Towards Development, Security and Human Rights for All”, Report of the Secretary-General, Documento ONU A/59/2005, 21 de marzo 2005.

21 Resolución del Consejo de Seguridad 1645 (2005), Documento ONU S/RES/1645 (2005), 20 de diciembre 2005, y Resolución de la Asamblea General 60/180 The Peacebuilding Commission, Documento ONU A/ RES/60/180, 30 de diciembre 2005. 
Las resoluciones reconocen "la responsabilidad primordial de gobiernos nacionales y transitorios y de las autoridades de países saliendo de conflicto o con riesgo de volver a caer en él, donde estén establecidas, de identificar sus prioridades y estrategias para la consolidación de la paz después del conflicto, con una perspectiva de aseguramiento de la propiedad nacional". También subrayan "la responsabilidad primordial del Concejo (de Seguridad) para el mantenimiento de la paz y seguridad internacional de acuerdo a los estatutos”. Estas dos responsabilidades no son contradictorias entre sí ni con la responsabilidad de la PBC, puesto que el sistema de responsabilidades deber coexistir con la soberanía del Estado. La PBC ayudará a los gobiernos de los Estados después del conflicto mientras estos no estén habilitados o no puedan ejercer sus poderes soberanos apropiadamente. Esta ayuda esta teóricamente entendida para ser alineada con la soberanía, como la consolidación de la paz simplemente ayuda al ejercicio de la soberanía. Pero cuando el gobierno de un Estado después de un conflicto esté aparentemente inhabilitado o incapacitado de llevar a cabo su responsabilidad, el Concejo de Seguridad será llamado a ejercer su responsabilidad internacional de tratar con él.

Se dieron muchas confrontaciones y fricciones entre los Estados miembros hasta que finalmente se lograron alcanzar los contenidos de las resoluciones para establecer la PBC. Va más allá del propósito de este documento examinar los detalles de estos debates. Es suficiente decir que mientras el grupo liderado por los Estados Unidos quería que la PBC fuera lo más efectiva posible bajo el comando del Concejo de Seguridad, una mayoría de países en desarrollo tenían prevenciones ante tal enfoque e hicieron algunos cambios significativos, incluyendo los roles principales del Concejo Económico y Social y de la Asamblea General. Aparte de estos detalles, la confrontación abarcó el gran mapa político de la dividida sociedad internacional. Había un grupo de Estados que querían que un órgano activo para la consolidación de la paz respondiera a las crisis de los Estados fallidos. El grupo de oposición acordó el carácter de intervención en la PBC y quiso establecer barreras para restringir sus actividades.

Teóricamente, esta confrontación puede ser explicada por la postura que implica la "responsabilidad de proteger". Aquellos que apoyan la RTP querían una PBC fuerte, proactiva y eficiente, a quienes les preocupa la RTP querían una PBC limitada. El resultado fue un acuerdo mutuo entre los dos grupos. Esto muestra el estatus de la teoría liberal de soberanía. Esta es una concepción amplia y compartida de soberanía. No podemos ignorar su impacto sobre la forma de pensar de la sociedad internacional. Si embargo, es cierto que un número considerable de Estados no están totalmente conformes con esta teoría de 
soberanía. La interpretación actual de la consolidación de la paz debe ser bien explicada por el estatus de la RTP y la teoría liberal de soberanía.

La consolidación de la paz o las operaciones de paz llevadas a cabo por la comunidad internacional probablemente continuarán evolucionando alrededor de la confrontación de estos dos grupos con diferentes perspectivas de soberanía. Habrá muchos más debates y acuerdos en muchas instancias relacionadas con la consolidación de la paz y la soberanía. Debemos mantener nuestra atención en la confrontación de los pros y los contras de la RTP. Sin embargo, no se puede negar que la teoría liberal de soberanía ha empezado en gran medida a definir las bases y el futuro de la consolidación de la paz .

\section{Conclusiones: reconsideración del nacionalismo, regionalismo y globalismo}

Este artículo ha argumentado que el nacionalismo, el regionalismo y el globalismo son asuntos interdisciplinarios que no son necesariamente contradictorios entre sí. Seguidamente se discutió que la concepción liberal de soberanía, definida como responsabilidad, constituye la base teórica de la consolidación de la paz en el mundo contemporáneo, la cual es un acto de intervención sin violar el principio de soberanía así definido. Al hacerlo así, el artículo ha sugerido que el cruce interdisciplinario de los tres elementos, el nacionalismo, el regionalismo y el globalismo, puede ser identificado en las actividades de consolidación de la paz caracterizadas por la concepción liberal de soberanía definida como responsabilidad.

A la consolidación de la paz le concierne el nacionalismo, dado que ésta tiene como objetivo instituir un Estado-nación estable con un gobierno nacional responsable. Un entendimiento sano del nacionalismo constituye la fundación de la consolidación de la paz en este sentido. Del mismo modo a la consolidación de la paz le concierne el regionalismo, dado que ésta tiene como objetivo establecer un marco regional para la paz en el que la sociedad en conflicto pueda ser absorbida. Se espera de un gobierno nacional responsable que construya relaciones estables con sus países vecinos; vecinos de los que a su vez se espera que atiendan a países de la región que estén pasando por una etapa de postconflicto. Asímismo, a la consolidación de la paz le concierne el globalismo, dado que ésta debe tratar con una multiplicidad de asuntos globales para solidificar la paz en una sociedad después de un conflicto. En primer lugar, la lógica de la consolidación de la paz se encuentra fundada sobre valores entre los que se incluye la responsabilidad de proteger. La concepción liberal de soberanía implica un carácter múltiple en relación con el nacionalismo, el regionalismo y el globalismo. La ICISS argumenta que: 
La soberanía aún importa. Es evidente que los Estados efectivos y legítimos continúan siendo la mejor manera de asegurar que los beneficios de la internacionalización del comercio, la inversión, la tecnología y la comunicación sean compartidos equitativamente. Aquellos Estados que puedan apelar a una fuerte alianza regional, a la paz interna y a una sociedad civil fuerte e independiente, parecen claramente estar en mejor posición para beneficiarse de la globalización. También serán éstos seguramente los más respetuosos de los derechos humanos. Y en términos de seguridad, es mucho más probable lograr un sistema internacional cohesivo y pacífico a través de la cooperación de Estados efectivos y seguros de su lugar en el mundo, que a través de un ambiente de entidades estatales frágiles, colapsadas, fragmentadas o caóticas. ${ }^{22}$

Para los estudiosos de los procesos de consolidación de la paz es importante tener presente que ésta es un asunto complejo que relaciona al nacionalismo, al regionalismo y al globalismo de diferentes maneras, en diferentes lugares y momentos. Por supuesto, la consolidación de la paz no es la única excepción. Los tres elementos de nacionalismo, regionalismo y globalismo se interrelacionan no solamente en la consolidación de la paz y la soberanía, sino también en otros diversos valores y movimientos políticos.

Lo que podemos sacar de esta observación es que el nacionalismo, el regionalismo y el globalismo ya no se repelen entre sí en el mundo actual. No es productivo sobreenfatizar las ventajas de uno de estos elementos para anular las desventajas de otro. Las posibilidades que brindan los múltiples conjuntos de sus combinaciones indican la infructuosidad de los intentos simplistas. Podríamos todavía referirnos a los tres elementos como herramientas analíticas para entender mejor realidades complejas. Lo que debemos hacer, entonces, es identificar las configuraciones de estos tres elementos cuando interactúan con otros valores y movimientos políticos de nuestro complejo mundo contemporáneo. 